\title{
The effects of postnatal health education for mothers on infant care and family planning practices in Nepal: a randomised controlled trial
}

\author{
Alison Bolam, Dharma S Manandhar, Purna Shrestha, Matthew Ellis, Anthony M de L Costello
}

\begin{abstract}
Objectives: To evaluate impact of postnatal health education for mothers on infant care and postnatal family planning practices in Nepal.

Design: Randomised controlled trial with community follow up at 3 and 6 months post partum by interview. Initial household survey of study areas to identify all pregnant women to facilitate follow up.

Setting: Main maternity hospital in Kathmandu, Nepal. Follow up in urban Kathmandu and a periurban area southwest of the city.

Subjects: 540 mothers randomly allocated to one of four groups: health education immediately after birth and three months later (group A), at birth only (group B), at three months only (group C), or none (group D).

Interventions: Structured baseline household questionnaire; 20 minute, one to one health education at birth and three months later.

Main outcome measures: Duration of exclusive breast feeding, appropriate immunisation of infant, knowledge of oral rehydration solution and need to continue breast feeding in diarrhoea, knowledge of infant signs suggesting pneumonia, uptake of postnatal family planning.

Results: Mothers in groups A and B (received health education at birth) were slightly more likely to use contraception at six months after birth compared with mothers in groups $\mathrm{C}$ and $\mathrm{D}$ (no health education at birth) (odds ratio 1.62, 95\% confidence interval 1.06 to 2.5 ). There were no other significant differences between groups with regards to infant feeding, infant care, or immunisation.

Conclusions: Our findings suggest that the recommended practice of individual health education for postnatal mothers in poor communities has no impact on infant feeding, care, or immunisation, although uptake of family planning may be slightly enhanced.
\end{abstract}

\section{Introduction}

The rational approach to health promotion-that information given by health workers during clinic based or community based contacts will bring about a change in health behaviour-is still an integral part of primary healthcare strategies. ${ }^{12}$ In practice, opportunities for one to one health education are given low priority by busy health workers. A survey of perinatal services across India reported that opportunities to give health education messages to mothers in the community were invariably missed. ${ }^{3}$

The effectiveness of health education has also been questioned. A recent review of over 500 articles about health education in developing countries found that only $11 \%$ described and evaluated actual attempts at health education. Of these, four described randomised studies and only three fulfilled the author's criteria for a rigorously designed evaluation. ${ }^{4}$ In countries with few resources there is also a trade off between impact and sustainability. Interventions that are considered successful usually result from small scale, well resourced projects which cannot be reproduced on a large scale. One non-randomised evaluation of an initiative to encourage postnatal health education in a district hospital in Bihar, India, did show significant improvements in early breast feeding practices, although health education by the health workers was not maintained in the longer term. ${ }^{5}$

With increasing use of hospital maternity and immunisation services, especially in urban areas of the developing world, ${ }^{6}$ perinatal contact with mothers represents an opportunity for health education about infant care and family planning. In developing countries 50-60\% of infant deaths occur in the neonatal period, ${ }^{7}$ and mortality from acute respiratory infections is highest in the first two months of life, when a mother's response to warning signs is crucial for survival. Failure to use postnatal contraception may also lead to an early repeat pregnancy, with attendant risks to maternal health.

In our study, the prospectively defined hypothesis was that one to one postnatal health education for mothers would positively affect their subsequent knowledge of and practices about infant care and family planning. Because an intervention should be feasible and sustainable on a large scale, education was restricted to a maximum of two contacts. Clinical objectives were to evaluate the impact of the intervention on uptake of immunisation, knowledge about and care of acute respiratory infections and diarrhoea in
Centre for

International Child Health, Institute of Child Health, London WC1N $1 \mathrm{EH}$

Alison Bolam, research fellow

Matthew Ellis, research fellow Anthony M de L Costello, reader in international child health

Mother and Infant Research Activities (MIRA), Maternity Hospital,

Kathmandu, c/o GPO Box 921, Kathmandu, Nepal Dharma S Manandhar, consultant paediatrician Purna Shrestha, research assistant

Correspondence to: Dr Costello a.costello@ich. ucl.ac.uk

BMJ 1998;316:805-11 
infants, the duration of exclusive breast feeding, infant growth, and use of postnatal family planning services.

\section{Subjects and methods}

The study was conducted from November 1994 to May 1996. Oral consent from mothers for inclusion in the study was obtained before assignment. The study received ethical approval from the Nepal Health Research Council.

\section{Setting}

Nepal is one of the least developed countries in the world, with an infant mortality of 98/1000 live births, maternal mortality of 1500/100 000 live births, $26 \%$ adult literacy, and a prevalence of contraceptive use of 23\%. The estimated population of Kathmandu municipality is 500000 , with an annual urban growth rate of $7.4 \% .^{9}$ Prasuti Griha is the main government funded maternity hospital in Kathmandu, with 250 beds, 15000 deliveries annually, and outpatient services for the local urban and surrounding populations. As there are no formal addresses in Kathmandu, a house to house survey of two communities was conducted before the study. Kirtipur is a periurban area $5 \mathrm{~km}$ south west of the hospital that contains 3663 households with a total population of 21368 . It is a settled community of mainly wage labourers and farmers. Kalimati is an urban area of central Kathmandu situated $2 \mathrm{~km}$ from Prasuti Griha and containing 2467 households with a total population of 13875 . This is a mixed community of long term residents and recent migrants.

\section{Eligibility}

All pregnant women admitted to Prasuti Griha hospital for delivery residing in these two communities were eligible for entry to the trial. Two mothers entered into

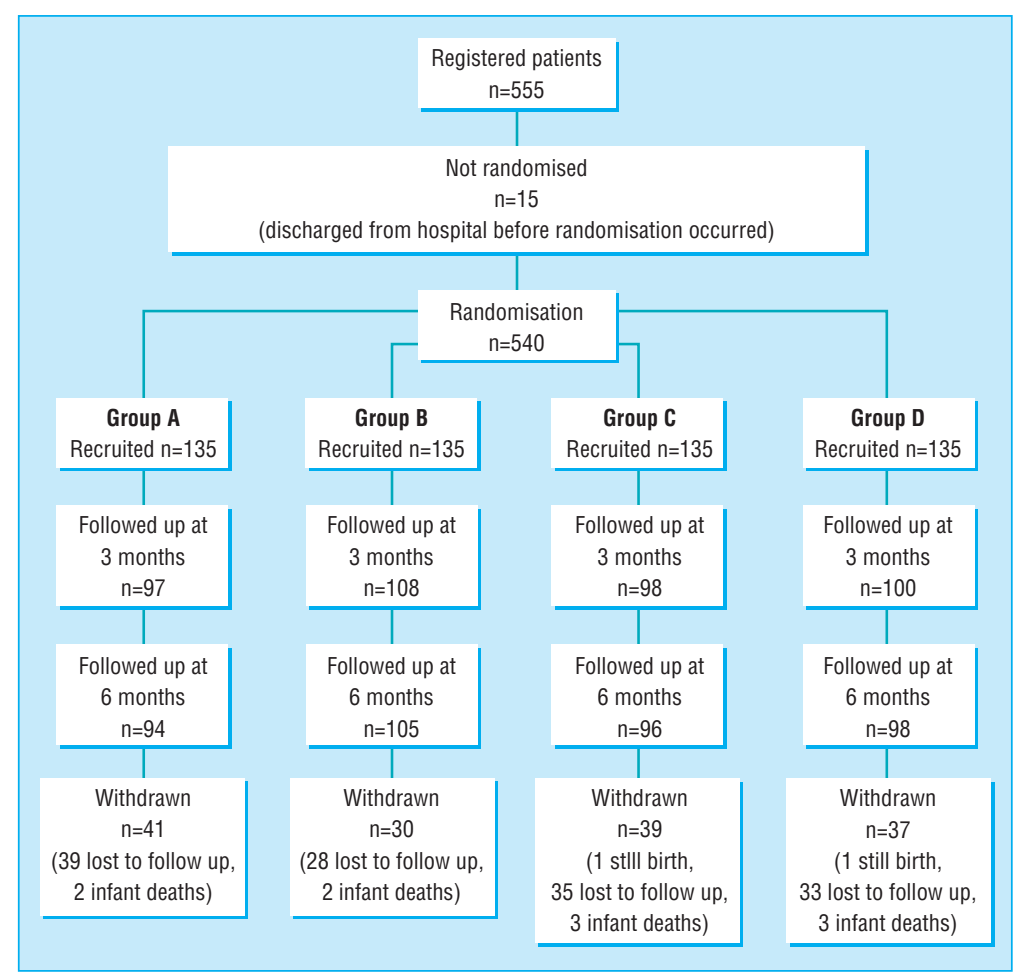

Flow chart showing progress of mothers through randomised trial the trial whose deliveries resulted in a stillbirth were withdrawn from the trial and received neither the intervention nor follow up.

\section{Protocol}

The health education intervention was developed with hospital staff in collaboration with consultants experienced in health education and women's development. Three female health educators, two midwives, and one community health worker were trained to give the health education. All were fluent in the two local languages, Nepali and Newari, and conducted the education intervention in the appropriate language. The health education session lasted about 20 minutes and was designed to be interactive and supportive rather than prescriptive in style. It was tested beforehand with 20 mothers, and modifications were made in the light of this experience. The health educators were monitored weekly during the trial by two principal investigators to check the quality of the intervention with regards to the content and the style of delivery, especially the level of interaction, and constructive critical feedback was given.

The first education session was conducted in a quiet room before discharge from the hospital. On average, seven mothers were enrolled in the trial each week from 250-300 admissions to the postnatal wards, so the risks of contamination (mothers in different groups sharing information) were negligible given that mothers were seen individually for the education intervention. The second education session was conducted in the mothers' home three months after delivery (mean length of time 14.1 (SD 2.4) weeks).

Although the health education given at birth and three months covered broadly the same areas, more emphasis was placed on the importance of exclusive breast feeding in the first session and on the need for family planning in the second session. The topics covered were infant feeding, treatment of diarrhoea, recognition of and response to symptoms suggesting acute respiratory infection in young infants, the importance of immunisation, and the importance of contraception after the puerperium. For each topic, the mothers were initially asked questions (such as, "How are you planning to feed your baby?" and "How would you know if your baby had pneumonia?") and given time to respond to encourage interaction. A discussion would follow depending on the response. For each topic, the discussion led on to the health educator giving key messages (see box) illustrated with large pictures on a cloth flip chart developed by local artists from health materials supplied by Unicef.

At the end of each session the health educator repeated the key messages covered and asked the mother if she had any other questions.

\section{Outcome measures}

After birth, data were collected on the pregnancy; mode and outcome of delivery; and infant gestational age, birth weight, length, and head circumference. Infant weight was measured to the nearest $50 \mathrm{~g}$ with a Soenhle electric infant weighing scale. Infant length was measured to the nearest $0.5 \mathrm{~cm}$ with a Rollametre (Child Growth Foundation), and head circumference was measured to the nearest $0.1 \mathrm{~cm}$ with a tape meas- 


\section{Key messages given by health educators}

Infant feeding-The advantages of breast feeding (it is clean, nutritious, prevents infection, helps family planning); the dangers of bottle feeding (risk of diarrhoea, reduction in mother's milk supply); how to increase the supply of breast milk (maternal diet, fluids, feeding soon after birth)

Diarrhoea-The dangers of diarrhoea; what to do if the infant develops diarrhoea (continue breast feeding, give oral rehydration solution, go to a health centre or doctor if diarrhoea persists)

Symptoms and response to acute respiratory infection -Visit a health worker if the infant develops cough, chest indrawing, fast breathing, or poor feeding

Immunisation - The importance of full immunisation; where to go for the first or subsequent injections Family planning-The importance of restarting contraception no later than 8 weeks after birth; the location of the nearest family planning clinic; the choice of methods; the availability of sterilisation services at the hospital

ure. Gestational age was assessed by the Parkin method. $^{10}$

Women were followed up at 3 and 6 months post partum in their homes, when data relating to our outcome measures were collected. Primary outcomes were the duration of exclusive breast feeding; mothers' knowledge of important signs of pneumonia and appropriate management of diarrhoea (mothers were asked: "How do you know if your baby with cough has pneumonia?" and, "If your baby has diarrhoea how must you care for him?"); uptake of immunisation; and use of postnatal family planning services. A secondary outcome was infant nutritional status.

\section{Sample size}

To assess the baseline situation we reviewed recent national survey data and conducted a pilot survey at the hospital outpatient clinic of 200 postnatal mothers -100 at birth and 100 at up to 4 months post partum.

Duration of exclusive breast feeding-The pilot survey showed 34\% mothers were exclusively breast feeding at 4 months post partum. These were well motivated women attending the hospital postnatally for infant immunisation. Another study of breast feeding practices in Nepal showed that only $20 \%$ of infants in Kathmandu were exclusively breast fed by 4 months of age. ${ }^{11}$ We hypothesised that $25 \%$ of mothers with no educational intervention would exclusively breast feed at 4 months, with an improvement to $40 \%$ in the intervention group: to detect this difference with $95 \%$ confidence limits and a power of $80 \%$, we needed to enter 165 mothers into each group.

Infant nutritional status-If education helps to prolong the duration of exclusive breast feeding, nutritional outcome might be improved. Assuming infants of mothers receiving the intervention grew on average along the 50th centile for British infants, a difference of $300 \mathrm{~g}$ in weight at 6 months $(3.8 \%)$ between the group receiving no education with the two groups receiving health education at birth would be detected with 95\% confidence limits and a power of $80 \%$ with sample sizes of 131 and 262.
Mothers' knowledge of managing infant diarrhoea and acute respiratory infection-A Nepal national survey in 1994 found that only $37 \%$ of urban children with diarrhoea received oral rehydration solution. ${ }^{12}$ In Pakistan few mothers spontaneously mentioned rapid breathing as a sign of pneumonia, ${ }^{13}$ and in the Philippines only $22 \%$ of cases of severe acute respiratory infection were recognised as severe by the mothers. ${ }^{14} \mathrm{We}$ hypothesised that $40 \%$ of control mothers and $60 \%$ of intervention mothers would correctly describe the signs of pneumonia and how to manage diarrhoea, requiring 107 mothers in each group (95\% confidence interval, power $80 \%$ ).

Immunisation uptake-In 1991 in Nepal 74\% of children aged 12 months had received three doses of diphtheria and pertussis vaccine and oral polio vaccine, and $81 \%$ were vaccinated against tuberculosis. ${ }^{15}$ We hypothesised that $40 \%$ of control and $60 \%$ of intervention infants would be fully immunised by 6 months of age, requiring 107 mothers in each group (95\% confidence interval, power 80\%).

Family planning - In our pilot survey $20 \%$ of postnatal mothers were using a method of contraception at 4 months. Use of contraceptives by currently married women nationally was estimated at $14 \%,{ }^{15}$ but this can be assumed to be higher in urban areas. We hypothesised a $20 \%$ uptake of contraception in the

Table 1 Baseline details of 540 mothers and infants recruited for study. Values are numbers (percentages) unless stated otherwise

\begin{tabular}{|c|c|c|c|c|c|}
\hline \multirow[b]{2}{*}{ Variable } & \multicolumn{4}{|c|}{ Intervention group* } & \multirow{2}{*}{$\begin{array}{c}\text { All } \\
\text { subjects } \\
(\mathrm{n}=540)\end{array}$} \\
\hline & $\begin{array}{l}\text { Group A } \\
(\mathrm{n}=135)\end{array}$ & $\begin{array}{l}\text { Group B } \\
(n=135)\end{array}$ & $\begin{array}{l}\text { Group C } \\
(\mathrm{n}=135)\end{array}$ & $\begin{array}{l}\text { Group D } \\
(n=135)\end{array}$ & \\
\hline \multicolumn{6}{|l|}{ Mothers } \\
\hline Mean (SD) age (years) $(n=538)$ & $23.4(4.0)$ & $23.2(4.3)$ & $23.1(4.2)$ & $24.0(3.4)$ & $23.4(4.0)$ \\
\hline \multicolumn{6}{|l|}{ Parity $(n=510):$} \\
\hline Primigravida & $61(50)$ & $65(50)$ & $77(60)$ & $68(53)$ & $271(53)$ \\
\hline Multigravida & $62(50)$ & $65(50)$ & $52(40)$ & $60(47)$ & $239(47)$ \\
\hline \multicolumn{6}{|l|}{ Area of living: } \\
\hline Periurban & $51(37)$ & $54(40)$ & $45(33)$ & $50(38)$ & $200(37)$ \\
\hline Urban & $84(63)$ & $81(60)$ & $90(67)$ & $85(62)$ & $340(63)$ \\
\hline \multicolumn{6}{|l|}{ Woman's occupation $(n=508)$ : } \\
\hline Housewife & $102(84)$ & $106(82)$ & $111(86)$ & $102(80)$ & $421(83)$ \\
\hline Other & $20(16)$ & $24(18)$ & $18(14)$ & $25(20)$ & $87(17)$ \\
\hline \multicolumn{6}{|l|}{ Woman's educational status $(n=509)$ : } \\
\hline Illiterate & $39(32)$ & $26(20)$ & $37(29)$ & $38(30)$ & $140(28)$ \\
\hline Literate up to primary level only & $26(21)$ & $38(30)$ & $31(15)$ & $27(21)$ & $122(24)$ \\
\hline Secondary level and above & $58(48)$ & $66(50)$ & $60(47)$ & $63(49)$ & $247(49)$ \\
\hline \multicolumn{6}{|l|}{ Husband's monthly income $(n=500)$ : } \\
\hline Low $(<\$ 20)$ & $6(5)$ & $10(8)$ & $6(5)$ & $3(2)$ & $25(5)$ \\
\hline Medium $(\$ 20-100)$ & $102(84)$ & $96(74)$ & $99(80)$ & $105(84)$ & $402(81)$ \\
\hline High $(>\$ 100)$ & $14(12)$ & $23(18)$ & $19(15)$ & $17(14)$ & $73(15)$ \\
\hline \multicolumn{6}{|l|}{ Delivery mode: } \\
\hline Normal & $122(90)$ & $118(87)$ & $111(82)$ & $105(78)$ & $456(84)$ \\
\hline $\begin{array}{l}\text { Assisted vaginal or caesarean } \\
\text { section }\end{array}$ & $13(10)$ & $17(13)$ & $24(18)$ & $30(22)$ & $85(16)$ \\
\hline
\end{tabular}

Infants

Sex:

\begin{tabular}{lccccc}
\hline Male & $75(55)$ & $60(45)$ & $63(47)$ & $67(49)$ & $265(49)$ \\
\hline Female & $60(45)$ & $75(55)$ & $72(53)$ & $68(51)$ & $275(51)$ \\
\hline Mean (SD) weight (kg) $(\mathrm{n}=533)$ & $2.75(0.4)$ & $2.75(0.4)$ & $2.78(0.5)$ & $2.67(0.5)$ & $2.74(0.5)$ \\
\hline Mean (SD) length (cm) (n=528) & $48.5(2.7)$ & $48.6(2.8)$ & $48.7(3.3)$ & $48.3(3.4)$ & $48.5(3.1)$ \\
\hline $\begin{array}{l}\text { Mean (SD) head circumference (cm) } \\
(\mathrm{n}=531)\end{array}$ & $33.6(1.3)$ & $33.8(2.3)$ & $33.8(1.9)$ & $33.9(2.9)$ & $33.8(2.2)$ \\
$\begin{array}{l}\text { Mean (SD) gestation (weeks) } \\
(\mathrm{n}=514)\end{array}$ & $39.3(2.2)$ & $39.4(1.7)$ & $39.4(1.8)$ & $39.1(2.4)$ & $39.3(2.0)$ \\
\hline
\end{tabular}

*Group $A=$ health education given immediately after birth and 3 months later; group $B=$ education given at birth only; group $\mathrm{C}=$ education given at 3 months only; group $\mathrm{D}=$ no education given. 
Table 2 Outcomes recorded at 3 months post partum for 403 mothers and infants. Values are numbers (percentages) unless stated otherwise

\begin{tabular}{|c|c|c|c|c|}
\hline \multirow[b]{2}{*}{ Variable } & \multicolumn{2}{|c|}{ Intervention group* } & \multirow[b]{2}{*}{ Odds ratio $(95 \% \mathrm{CI})$} & \multirow[b]{2}{*}{$P$ value } \\
\hline & $\begin{array}{c}\text { Groups A } \\
\text { and B }\end{array}$ & $\begin{array}{c}\text { Groups C } \\
\text { and D }\end{array}$ & & \\
\hline \multicolumn{5}{|l|}{ Infant feeding practice: } \\
\hline Exclusive breast feeding & $120(59)$ & $117(59)$ & \multirow{2}{*}{1.00 (0.67 to 1.49$)$} & \multirow{2}{*}{1.00} \\
\hline Other & $84(41)$ & $82(41)$ & & \\
\hline Mean (SD) weight $(\mathrm{kg})$ & $5.7(0.85)$ & $5.6(0.9)$ & $\begin{array}{c}\text { Mean difference } 0.100 \\
(-0.072 \text { to } 0.271)\end{array}$ & 0.25 \\
\hline Mean (SD) length (cm) & $58.4(4.6)$ & $58.5(6.2)$ & $\begin{array}{c}\text { Mean difference }-0.041 \\
\quad(-1.11 \text { to } 1.03)\end{array}$ & 0.94 \\
\hline Mean (SD) head circumference $(\mathrm{cm})$ & $40.9(2.7)$ & $40.7(4.1)$ & $\begin{array}{l}\text { Mean difference } 0.217 \\
(-0.469 \text { to } 0.902)\end{array}$ & 0.53 \\
\hline \multicolumn{5}{|l|}{ Immunisation: } \\
\hline Appropriate† & $179(87)$ & $169(85)$ & \multirow{2}{*}{1.18 (0.67 to 2.08$)$} & \multirow{2}{*}{0.66} \\
\hline Other & $26(13)$ & $29(15)$ & & \\
\hline \multicolumn{5}{|l|}{ Knowledge of signs of pneumonia: } \\
\hline \multicolumn{5}{|l|}{ Indrawing: } \\
\hline Yes & $54(26)$ & $44(22)$ & \multirow{2}{*}{1.25 (0.79 to 1.97$)$} & \multirow{2}{*}{0.35} \\
\hline No & $151(74)$ & $154(78)$ & & \\
\hline \multicolumn{5}{|l|}{ Tachypnoea: } \\
\hline Yes & $107(52)$ & $84(42)$ & \multirow{2}{*}{1.48 (1.00 to 2.19$)$} & \multirow{2}{*}{0.06} \\
\hline No & $98(48)$ & 114 (58) & & \\
\hline
\end{tabular}

In case of diarrhoea knows to:

\begin{tabular}{|c|c|c|c|c|}
\hline Contin & & & & \\
\hline Yes & $94(46)$ & $94(47)$ & \multirow{2}{*}{0.95 (0.64 to 1.40$)$} & \multirow{2}{*}{0.84} \\
\hline No & $111(54)$ & $105(53)$ & & \\
\hline \multicolumn{5}{|c|}{ Give oral rehydration solution: } \\
\hline Yes & $177(86)$ & $165(83)$ & \multirow{2}{*}{$1.26(0.74$ to 2.17$)$} & \multirow{2}{*}{0.41} \\
\hline No & $28(14)$ & $33(17)$ & & \\
\hline
\end{tabular}

\begin{tabular}{lrrrrr}
\hline \multicolumn{4}{l}{ Any contraceptive used for family planning: } & & \\
\hline No & $163(80)$ & $171(86)$ & $1.49(0.87$ to 2.53) & 0.14 \\
\cline { 1 - 3 } Yes & $40(20)$ & $28(14)$ & &
\end{tabular}

*Groups $A$ and $B=$ health education given at birth; groups $C$ and $D=$ no health education at birth.

$\dagger B C G$ plus at least two doses of diphtheria and pertussis vaccine and oral polio vaccine.

control group by 6 months post partum and an improvement to $33 \%$ in the intervention group, requiring 195 mothers in each group $(95 \%$ confidence interval, power $80 \%$ ).

Using these figures, we enrolled 540 subjects in order to compare four subgroups: mothers receiving health education immediately after birth and at 3 months post partum (group A), health education at birth only (group B), health education at three months only (group C), or no health education at all (group D). For outcomes at three months, we combined groups A and $\mathrm{B}$ as the intervention group and $\mathrm{C}$ and $\mathrm{D}$ as the control group. For the outcomes at 6 months, the groups were compared individually.

\section{Randomisation and blinding}

The unit of randomisation was the individual mother. Restricted randomisation was used in blocks of 20, each block consisting of a random ordering of the numbers 0-19. Numbers 0-4, 5-9, 10-14, and 15-19 were assigned to groups A to $\mathrm{D}$ respectively. The details of allocation to groups for consecutively recruited mothers were in sealed envelopes. Timing of assignment was when a mother was identified by the research team either in labour or shortly after delivery. A member of the research team checked the hospital admission register at least twice each day between 7 am and $8 \mathrm{pm}$. The generator of the assignment was not involved in the execution of the allocation. There were no prospectively defined rules for stopping the trial.
Clearly, the mothers recruited and the health educators were not blind to the assignment of mothers to different groups. The outcome assessors were always blind to the assignment at both the 3 and 6 month follow up visits. Staff who were involved in data collection at the 3 month follow up were not involved in data collection at 6 months. The data analysts were not blind to the coding of the groups.

\section{Statistical analysis}

To estimate the effect of the trial intervention between the groups we measured the mean differences, 95\% confidence intervals, and $\mathrm{P}$ values for continuous data, and the odds ratios, 95\% confidence intervals, and $\mathrm{P}$ values for categorical data. We used the MantelHaenszel test to check for heterogeneity of categorical data, giving $\chi^{2}$ and $\mathrm{P}$ values, and analysis of variance for continuous data, giving $F$ values and $\mathrm{P}$ values. We analysed data on an intention to treat basis in which we compared intervention and control groups irrespective of the quality of the education intervention. For statistical analysis, we used computer software Statview version 4.0 and Stata version 5.0.

\section{Results}

Subjects

The figure shows the details of participant flow and follow up. We recruited 540 mothers, 135 to each of the four groups, and followed up 403 (75\%) to 3 months post partum and $393(73 \%)$ to 6 months. The main reason for loss to follow up was the mother moving back to her parental home as part of cultural tradition. Table 1 shows the baseline characteristics of the mothers and infants.

Mortality-There were no maternal deaths, two stillbirths, and 10 infant deaths. Mothers whose infants were stillborn were withdrawn from the study. All the infant deaths occurred in the neonatal period: two occurred in group A, two in group B, three in group C, and three in group D. Seven of these infants were born prematurely and had a birth weight less than $2.5 \mathrm{~kg}$, two had severe congenital abnormalities, and one died from acute respiratory infection at home at 4 weeks of age.

\section{Outcome at 3 months}

Table 2 shows the outcomes at 3 months post partum. We compared mothers in groups $\mathrm{A}$ and $\mathrm{B}$, who received health education at birth, with those in groups $\mathrm{C}$ and $\mathrm{D}$, who received none. Mothers in groups $\mathrm{A}$ and B were slightly more likely to report tachypnoea as a sign of acute respiratory infection, but this did not quite reach statistical significance (odds ratio 1.48, 95\% confidence interval 1.00 to $2.19, \mathrm{P}=0.06$ ). Also, $20 \%$ of mothers in groups $\mathrm{A}$ and $\mathrm{B}$ were using contraception compared with only $14 \%$ of those in groups C and D, but this difference was not significant. There were no differences for the other outcomes.

Immunisation coverage was higher than we had hypothesised for both groups (85\% in groups C and D, $87 \%$ in groups A and B): our sample size would have detected an increase to $93 \%$ coverage in groups A and $\mathrm{B}$ at $5 \%$ significance (one sided test) and $78 \%$ power . 
Table 3 Outcomes recorded at 6 months post partum for 393 mothers and infants. Values are numbers (percentages) unless stated otherwise

\begin{tabular}{|c|c|c|c|c|c|c|c|c|c|}
\hline \multirow[b]{2}{*}{ Variable } & \multicolumn{4}{|c|}{ Intervention group* } & \multicolumn{2}{|c|}{ Groups $A$ and $B v C$ and $D$} & \multicolumn{2}{|c|}{ Groups $A$ and $C v B$ and $D$} & \multirow{2}{*}{$\begin{array}{c}\text { Outcome by health } \\
\text { education at birth stratified } \\
\text { by health education at } 3 \\
\text { months }\end{array}$} \\
\hline & Group A & Group B & Group C & Group D & $\begin{array}{l}\text { Odds ratio } \\
(95 \% \mathrm{CI})\end{array}$ & $P$ value & $\begin{array}{l}\text { Odds ratio } \\
(95 \% \mathrm{Cl})\end{array}$ & $P$ value & \\
\hline \multicolumn{10}{|l|}{$\begin{array}{l}\text { Duration of exclusive breast feeding } \\
(\mathrm{n}=390) \text { : }\end{array}$} \\
\hline$\geqslant 5$ months & $31(33)$ & $25(24)$ & $27(29)$ & $27(28)$ & \multirow{2}{*}{$\begin{array}{c}1.01 \\
(0.65 \text { to } 1.56)\end{array}$} & \multirow[t]{2}{*}{1.00} & \multirow{2}{*}{$\begin{array}{c}1.29 \\
(0.83 \text { to } 2.0)\end{array}$} & \multirow[t]{2}{*}{0.31} & \multirow{2}{*}{$\begin{array}{l}\text { Mantel-Haenszel test: } \\
\chi^{2}=0.72, P=0.395\end{array}$} \\
\hline$<5$ months & $63(67)$ & $79(76)$ & $67(71)$ & $71(72)$ & & & & & \\
\hline Mean (SD) weight (kg) & $7.2(0.9)$ & $7.3(1.0)$ & $7.2(0.93)$ & $7.2(1.1)$ & $\begin{array}{c}\text { Mean difference } \\
0.028 \\
(-0.169 \text { to } 0.225)\end{array}$ & 0.78 & $\begin{array}{c}\text { Mean difference } \\
-0.06 \\
(-0.255 \text { to } \\
0.139)\end{array}$ & 0.56 & $\begin{array}{c}\text { Analysis of variance: } \\
\text { Education at } 3 \text { months } \\
F=0.085, P=0.77 \\
\text { No education } \\
F=0.01, P=0.92\end{array}$ \\
\hline Mean (SD) length (cm) & $62.4(7.4)$ & $63.2(4.0)$ & $62.3(7.0)$ & $62.9(4.5)$ & $\begin{array}{c}\text { Mean difference } \\
0.223 \\
(-0.95 \text { to } 1.39)\end{array}$ & 0.71 & $\begin{array}{c}\text { Mean difference } \\
-0.7 \\
(-1.87 \text { to } 0.47)\end{array}$ & 0.24 & $\begin{array}{c}\text { Analysis of variance: } \\
\text { Education at } 3 \text { months } \\
F=0.006, P=0.94 \\
\text { No education } \\
F=0.28, P=0.60\end{array}$ \\
\hline Mean (SD) head circumference $(\mathrm{cm})$ & $42.5(1.5)$ & $42.4(2.0)$ & $42.5(1.4)$ & $42.6(2.1)$ & $\begin{array}{c}\text { Mean difference } \\
-0.06 \\
(-0.41 \text { to } 0.29)\end{array}$ & 0.74 & $\begin{array}{c}\text { Mean difference } \\
-0.32 \\
(-0.39 \text { to } 0.32)\end{array}$ & 0.86 & $\begin{array}{c}\text { Analysis of variance: } \\
\text { Education at } 3 \text { months } \\
F=0.008, P=0.93 \\
\text { No education } \\
F=0.23, P=0.63\end{array}$ \\
\hline \multicolumn{10}{|l|}{ Immunisation: } \\
\hline Appropriate† & 90 (95) & $100(96)$ & $90(93)$ & $91(94)$ & \multirow{2}{*}{$\begin{array}{c}1.52 \\
(0.65 \text { to } 3.55)\end{array}$} & \multirow[t]{2}{*}{0.39} & 0.79 & \multirow[t]{2}{*}{0.66} & \multirow{2}{*}{$\begin{array}{l}\text { Mantel-Haenszel test: } \\
\chi^{2}=0.033, P=0.855\end{array}$} \\
\hline Other & $5(5)$ & $4(4)$ & $7(7)$ & $6(6)$ & & & (0.34 to 1.82$)$ & & \\
\hline \multicolumn{10}{|l|}{ Knowledge of signs of pneumonia: } \\
\hline \multicolumn{10}{|l|}{ Indrawing: } \\
\hline Yes & $27(28)$ & $26(25)$ & $26(27)$ & $20(21)$ & \multirow{2}{*}{$\begin{array}{c}1.17 \\
(0.75 \text { to } 1.84)\end{array}$} & \multirow[t]{2}{*}{0.56} & 1.28 & \multirow[t]{2}{*}{0.30} & \multirow{2}{*}{$\begin{array}{c}\text { Mantel-Haenszel test: } \\
\chi^{2}=0.13, P=0.72\end{array}$} \\
\hline No & $68(72)$ & $78(75)$ & $71(73)$ & $77(79)$ & & & (0.82 to 2.02$)$ & & \\
\hline \multicolumn{10}{|l|}{ Tachypnoea: } \\
\hline Yes & $51(54)$ & $56(54)$ & $56(58)$ & $44(45)$ & \multirow{2}{*}{$\begin{array}{c}1.09 \\
(0.74 \text { to } 1.62)\end{array}$} & \multirow[t]{2}{*}{0.69} & 1.27 & \multirow[t]{2}{*}{0.27} & \multirow{2}{*}{$\begin{array}{c}\text { Mantel-Haenszel test: } \\
\chi^{2}=1.54, P=0.21\end{array}$} \\
\hline No & $44(46)$ & $48(46)$ & $41(42)$ & $53(55)$ & & & (0.86 to 1.89$)$ & & \\
\hline \multicolumn{10}{|l|}{ In case of diarrhoea knows to: } \\
\hline \multicolumn{10}{|l|}{ Continue breast feeding: } \\
\hline Yes & $50(53)$ & $51(49)$ & $49(51)$ & $48(49)$ & \multirow{2}{*}{$\begin{array}{c}1.03 \\
(0.69 \text { to } 1.53)\end{array}$} & 0.92 & 1.10 & 0.69 & Mantel-Haenszel test: \\
\hline No & $45(47)$ & $53(51)$ & $48(49)$ & $49(51)$ & & & (0.74 to 1.63$)$ & & $\chi^{2}=0.064, P=0.799$ \\
\hline Give oral rehydration solution: & & & & & & & & & \\
\hline Yes & $89(94)$ & $96(92)$ & $94(97)$ & $90(93)$ & 0.72 & 0.53 & 1.64 & 0.3 & Mantel-Haenszel test: \\
\hline No & $6(6)$ & $8(8)$ & $3(3)$ & $7(7)$ & (0.32 to 1.63 ) & & (0.71 to 3.76$)$ & & $\chi^{2}=0.568, P=0.45$ \\
\hline Any contraceptive used for family plar & & & & & & & & & \\
\hline No & $62(65)$ & $64(62)$ & $72(74)$ & $71(73)$ & 1.62 & 0.03 & 0.86 & 0.59 & Mantel-Haenszel test: \\
\hline Yes & $33(35)$ & $40(38)$ & $25(26)$ & $26(27)$ & (1.06 to 2.50 ) & & (0.58 to 1.35$)$ & & $\chi^{2}=0.06, P=0.81$ \\
\hline
\end{tabular}

*Group $A=$ health education given immediately after birth and at 3 months later; group $B=$ education given at birth only; group $C=$ =education given at 3 months only; group $D=$ no education given. †BCG plus three doses of diphtheria and pertussis vaccine and oral polio vaccine.

\section{Outcome at 6 months}

Table 3 shows the outcomes at 6 months post partum. We made two broad comparisons: groups A and B (health education at birth) compared with groups $\mathrm{C}$ and $\mathrm{D}$ (no health education at birth), and groups $\mathrm{A}$ and C (health education at 3 months) compared with groups $\mathrm{B}$ and $\mathrm{D}$ (no health education at 3 months). The only significant difference we observed for all outcomes was an increase in uptake of family planning at 6 months in groups A and B (odds ratio 1.62, 95\% confidence interval 1.06 to 2.5 ). To test for interactions, we compared outcomes by health education at birth stratified by whether health education was given at 3 months post partum using tests for heterogeneity: we found no significant interactions.

Poststudy calculations of the power of our study to detect a significant, one sided difference in exclusive breast feeding between groups (based on our hypothesis of $25 \%$ in mothers given no health education and $40 \%$ in those given education) were $67 \%$ (comparing group A with group D) and 84\% (comparing groups A, B, and C with group D).

\section{Discussion}

This trial in Nepal has shown that a health education intervention (one to one counselling of mothers by health educators) given on two occasions, immediately after delivery and 3 months later, had no significant impact on the mothers' knowledge and practices of child care or infant health outcomes, but there was a slight improvement in uptake of family planning at 6 months after birth. Given the higher than expected level of immunisation in all groups, we cannot rule out the possibility that health education may have had an impact in situations where coverage is lower.

\section{Trial design}

Our study included only women who chose institutional delivery. Whether mothers who gave birth at home would benefit from health education more than those who gave birth at hospital is questionable, but it is difficult to target mothers delivering at home and to conduct a trial of intervention in the home.

The overall lack of impact on practices in infant care might also be explained by the length and frequency of the intervention. Our study deliberately 
involved a maximum of only two contacts with each mother in an attempt to evaluate a less intensive, more sustainable intervention. There is some evidence that health education at an individual level has an impact if messages are repeated frequently to patients, ${ }^{4}$ but multiple contact with patients in the community is difficult to sustain in a resource poor country such as Nepal. Pilot studies that report success are usually from well funded, small scale, non-government projects. ${ }^{16}$

A combination of antenatal and perinatal contacts might be more successful. In this trial $88 \%$ of women had attended one or more antenatal clinic appointments, at which only $3 \%$ had received any health education. Follow up rates for the trial were less than ideal $(75 \%$ at 3 months and $73 \%$ at 6 months) but reasonable for a trial conducted in difficult field conditions, where mothers often return to their parental home postnatally.

\section{Evaluation of health education interventions}

Recommendations for the design of health education interventions and the importance of including evaluation in health education programmes have been widely reported. ${ }^{47-20}$ For example, the American Public Health Association stated that "from the outset, a health promotion program should be organised, planned and implemented in such a way that its operation and effects can be evaluated." ${ }^{17}$ In practice, however, evaluation is rare. In a review of health education in developing countries spanning 10 years, only $11 \%$ of published articles described and evaluated the health education programme. ${ }^{4}$ Most of the evaluations were methodologically unsound so firm conclusions could not be drawn about the overall efficacy of health education. Randomised controlled studies, the ideal design, have rarely been reported from developing countries: only four of the studies reviewed by Loevinsohn used a randomised controlled design, and one of these failed to meet other of his criteria for an adequate study design. ${ }^{4}$

Health education by health workers is still seen as an important part of primary health care despite this lack of evidence of efficacy. Training of health and field workers to convey messages, and the development of health education materials, consumes a substantial proportion of health budgets in resource poor countries. Our negative findings suggest that much of this investment may be ineffective. Social cognitive theory, by contrast, suggests that experience from interactions within family, peer groups, or communities, rather than information per se, is the key to successful health promotion..$^{22}$

It might be argued that a postnatal health intervention would be more effective if it focuses on only one outcome. The small but significant increase in contraceptive use at 6 months post partum by the mothers receiving health education immediately after birth might have been even greater if this was the only subject discussed. This requires further evaluation because postnatal family planning and birth spacing have health benefits for both mothers and infants. It might also be argued that mothers in Nepal do not perceive many health workers as purveyors of credible knowledge about motherhood. In our study we deliberately selected health educators who were able to gain the respect of mothers through their experience
Key messages

- Health education is widely promoted in primary care, but there have been few rigorous evaluations of its impact, especially in developing countries

- A randomised controlled trial of postnatal individual health education for mothers given by trained female health workers showed no significant impact on maternal knowledge and practices of child care or on infant health outcomes, but there was a small improvement in uptake of family planning at six months after birth

- The efficacy of health education interventions that rely solely on giving people information to bring about a change in health behaviour is unproved; interventions should be evaluated before being implemented on a large scale

- Alternative strategies for health promotion in developing countries such as interactions within families, peer groups, or communities may be more effective but are costly and difficult to implement on a large scale

as midwives or community health workers, but who were also able to put mothers at ease during the education session.

\section{Conclusions}

Our results indicate the need for further, well designed evaluations of health education interventions that are randomised and controlled, provide a clear definition of aims, and present pre-intervention and postintervention data for carefully defined outcome measures. Future evaluations of education interventions also need to explore, through qualitative research, the understanding of the recipients and their reaction to the messages. It might be that behaviour can be changed in response to simple messages repeated frequently in many forums, but in developing countries there will a trade off between efficacy and cost: repeated home visits by friendly health workers may not be feasible on a large scale. It might also be the case that the desired changes in behaviour are not realistic for the individual or community because of economic, social, and cultural barriers. Interventions aimed at women must take into account their heavy workload in the home and field and their degree of influence within the household on decisions about child care, family planning, and health seeking behaviour.

We thank Dr Kasturi Malla for helpful discussions and the staff of Prasuti Griha and MIRA, Nepal, for their support. We are grateful to Diana Gibb, Ruth Gilbert, and Sally Kerry for advice on trial design and statistical analysis.

Contributors: $\mathrm{AB}$ coordinated the formulation of the trial design and protocol, supervised the trial implementation, and participated in data collection and analysis and writing of the paper. DSM helped to formulate the initial study hypothesis, trial design, and protocol; assisted with study implementation; and reviewed drafts of the paper. PS contributed to the study design, participated in data collection, and reviewed drafts of the paper. ME contributed to formulation of the trial design and protocol, and participated in data analysis and writing of the paper. AMdeLC conceived of the study hypothesis, developed the trial design and protocol, and contributed to data analysis 
and writing of the paper. $\mathrm{AB}$ and AMdeLC are guarantors for the paper.

Funding: Research grant from Britain's Department for International Development.

Conflict of interest: None.

World Health Organisation. The Alma Ata declaration. Geneva: WHO, 1978.

2 World Bank. Investing in health. The world development report. Oxford: Oxford University Press, 1993.

3 Bhargava SK, Singh KK, Saxena BN, eds. A national collaborative study of identification of high risk families, mothers and outcome of their offsprings with particular reference to the problem of maternal nutrition, low birth weight, perinatal and infant morbidity and mortality in rural and urban slum communities. New Delhi: Indian Council of Medical Research, 1990.

4 Loevinsohn BP. Health education in developing countries: a methodological review of published articles. Int J Epidemiol 1990;19: 788-94.

5 Prasad B, Costello AM de L. Impact and sustainability of a "baby friendly" health education intervention at a district hospital in Bihar, India. BMJ 1995;310:621-3

6 Bolam A, Manandhar DS, Shrestha P, Manandhar B, Ellis M, Costello AM. Maternity care utilisation in the Kathmandu Valley: a community based study. J Nepal Med Assoc 1997;35:122-9.

7 Ashworth A, Waterlow JC. Infant mortality in developing countries. Arch Dis Child 1982;57:882-4

8 Human Development Report. Published for the United Nations Development Programme. Oxford: Oxford University Press, 1996.

9 World Bank. World development report 1990. Washington DC: World Bank, 1990

10 Parkin JM, Hey EN, Clowes JS. Rapid assessment of gestational age in the newborn infant. Arch Dis Child 1976;51:259.
11 Neupane S. A study on breast feeding status in rural and urban areas of central development region, Nepal. Kathmandu: Unicef, 1992.

12 Nepal multiple indicator surveillance: preliminary report. Kathmandu: National Planning Commission, His Majesty's Government of Nepal, 1995.

13 Malik Kundi MZ, Anjum M, Mull DS, Mull JD. Maternal perceptions of pneumonia and pneumonia signs in Pakistani children. Soc Sci Med 1993;37:649-60.

14 Tupasi TE, Miguel CA, Tallo VL, Bagasao TMP, Natividad JN, Valencia $\mathrm{LB}$, et al. Child care practices of mothers: implications for intervention in acute respiratory infections. Ann Tropical Paediatrics 1989;9:82-8.

15 United Nations Children's Fund. The state of the world's children 1993. Oxford: Oxford University Press, 1993.

16 Kafatos A, Tsitoura S, Pantelakis S, Doxiadis S. Maternal and infant health education in a rural Greek community. Hygie 1991;10:32-7.

17 American Public Health Association Work Group on Health Promotion/ Disease Prevention. Criteria for the development of health promotion and education programs. Am J Public Health 1987;77:89-92.

18 Hubley JH. Barriers to health education in developing countries. Health Educ Res 1986;1:233-45.

19 Oakley A, Fullerton D, Holland J, Arnold S, France-Dawson M, Kelley P, et al. Sexual health education interventions for young people: a methodological review BMJ 1995;310:158-62.

20 Gyorkos TW, Tannenbaum TN, Abrahamowicz M, Oxman AD, Scott EA, Millson ME, et al. An approach to the development of practice guidelines for community health interventions. Can J Public Health 1994;85:S8-13.

21 Maibach E, Murphy DA. Self-efficacy in health promotion research and practice: conceptualisation and measurement. Health Educ Res 1995; 10:37-50.

22 Bandura A. Self-efficacy: toward a unifying theory of behavioural change. Psychol Rev 1977;84:191-215.

(Accepted 6 February 1998)

\section{Meta-analysis of short term low dose prednisolone versus placebo and non-steroidal anti-inflammatory drugs in rheumatoid arthritis}

Peter C Gøtzsche, Helle Krogh Johansen

\begin{abstract}
Objective: To determine whether short term, oral low dose prednisolone ( $\leqslant 15 \mathrm{mg}$ daily) is superior to placebo and non-steroidal anti-inflammatory drugs in patients with rheumatoid arthritis.

Design: Meta-analysis of randomised trials of oral corticosteroids compared with placebo or a non-steroidal anti-inflammatory drug.

Setting: Trials conducted anywhere in the world.

Subjects: Patients with rheumatoid arthritis.

Main outcome measures: Joint tenderness, pain, and grip strength. Outcomes measured on different scales were combined by using the standardised effect size (difference in effect divided by SD of the measurements).

Results: Ten studies were included in the meta-analysis. Prednisolone had a marked effect over placebo on joint tenderness (standardised effect size $1.31 ; 95 \%$ confidence interval 0.78 to 1.83 ), pain (1.75; 0.87 to 2.64$)$, and grip strength $(0.41 ; 0.13$ to 0.69$)$. Measured in the original units the differences were 12 (6 to 18) tender joints and $22 \mathrm{~mm} \mathrm{Hg} \mathrm{(} 5 \mathrm{~mm} \mathrm{Hg}$ to $40 \mathrm{~mm} \mathrm{Hg}$ ) for grip strength. Prednisolone also had a greater effect than non-steroidal anti-inflammatory drugs on joint tenderness $(0.63 ; 0.11$ to 1.16$)$ and pain (1.25; 0.26 to 2.24$)$, whereas the difference in grip strength was not significant $(0.31 ;-0.02$ to 0.64$)$. Measured in the original units the differences were 9
\end{abstract}

(5 to 12 ) tender joints and $12 \mathrm{~mm} \mathrm{Hg} \mathrm{(}-6 \mathrm{~mm} \mathrm{Hg}$ to $31 \mathrm{~mm} \mathrm{Hg}$ ). The risk of adverse effects during moderate and long term use seemed acceptable. Conclusion: Prednisolone in low doses $(\leqslant 15 \mathrm{mg}$ daily) may be used intermittently in patients with rheumatoid arthritis, particularly if the disease cannot be controlled by other means.

\section{Introduction}

Corticosteroids were first shown to be effective in patients with rheumatoid arthritis in 1949 in an uncontrolled study. ${ }^{1}$ In 1959, a two year randomised trial showed that an initial dose of prednisolone $20 \mathrm{mg}$ daily was significantly superior to aspirin $6 \mathrm{~g}$ daily. ${ }^{2}$ Important adverse effects were also noted, however, and the authors concluded that the highest acceptable dose for long term treatment was probably in the region of $10 \mathrm{mg}$ daily.

Corticosteroids have received renewed interest in recent years because of their possible beneficial effect on radiological progression. ${ }^{3}$ Tendencies towards such an effect were noted both in the early trials and in a recent report. ${ }^{4}$

These findings are interesting, but oral corticosteroids are still being used mainly for their symptomatic effect-for example, for acute exacerbations of rheumatoid arthritis and as "bridge therapy" before slow acting drugs have taken effect. ${ }^{5}$ The effect of low doses
Editorial by

Dennison and Cooper

Nordic Cochrane

Centre,

Rigshospitalet, Department 7112

Tagensvej $18 \mathrm{~B}$,

DK-2200

Copenhagen $\mathrm{N}$

Denmark

Peter C Gøtzsche, director

Helle Krogh

Johansen,

senior researcher

Correspondence to: Dr Gøtzsche p.c.gotzsche@ cochrane.dk

BMJ 1998;316:811-8 